\title{
Space Mobile Network Concepts for Missions Beyond Low Earth Orbit
}

\author{
David J. Israel, ${ }^{1}$ Christopher J. Roberts, ${ }^{2}$ and Robert M. Morgenstern ${ }^{3}$ \\ NASA Goddard Space Flight Center, Greenbelt, MD, 20771, USA \\ Jay L. Gao ${ }^{4}$ and Wallace S. Tai ${ }^{5}$ \\ Jet Propulsion Laboratory, California Institute of Technology, Pasadena, CA, 91109, USA
}

The Space Mobile Network (SMN) is an architectural framework that will allow for quicker, more efficient and more easily available space communications services, providing user spacecraft with an experience similar to that of terrestrial mobile network users. While previous papers have described SMN concept using examples of users in low-Earth orbit, the framework can also be applied beyond the near-Earth environment.

This paper details how SMN concepts such as user-initiated services, which will enable users to request access to high-performance link resources in response to real-time science or operational events, would be applied in and beyond the near-Earth regime. Specifically, the paper explores the application of user-initiated services to direct-to-Earth (DTE), relay, and DTE/relay hybrid scenarios in near-Earth, lunar, martian and other space regimes.

\section{Introduction}

NASA has introduced a framework, the Space Mobile Network (or SMN) [1][2], for a space communications and navigation architecture. SMN involves a set of architectural ideas that will allow for automated, more efficient and more easily available communications services, providing user spacecraft with increased network performance supportive of dynamic and autonomous mission scenarios. Users will be able to request communications services as needed from a network of diverse providers, including government and commercial providers. In this automatic and more accommodating system, user platforms could request services directly to tailor to their real-time needs; thus, SMN offers autonomous variable data collection without requiring pre-scheduled, manual requests, enabling users to obtain higher or lower data rates as needed. SMN also allows for improved availability and mitigation of delays in service provision by taking into account specific latency requirements.

While previous papers describing SMN concepts and implementation have typically demonstrated the ideas using case examples of users in low-Earth orbit (LEO), the concept is not restricted to LEO users and could be applied beyond LEO. This paper will describe SMN application scenarios for users at the Moon, the Sun-Earth Lagrange points and Mars, and will propose examples of how to implement those concepts. The concepts will include support through both direct-to-Earth links and space relays. This paper will also discuss how missions moving from beyond LEO will be able to continue under the same operations concepts, though implementation solutions may be different for the different scenarios.

\footnotetext{
${ }^{1}$ Architect, Exploration and Space Communications Projects Division, AIAA Senior Member

${ }^{2}$ Mission Manager, Exploration and Space Communications Projects Division, AIAA Member

${ }^{3}$ Systems Engineer, Exploration and Space Communications Projects Division

${ }^{4}$ Senior Researcher, Communications Architecture and Research Section

${ }^{5}$ Chief Engineer, Interplanetary Network Directorate
} 


\section{Space Mobile Network Concepts}

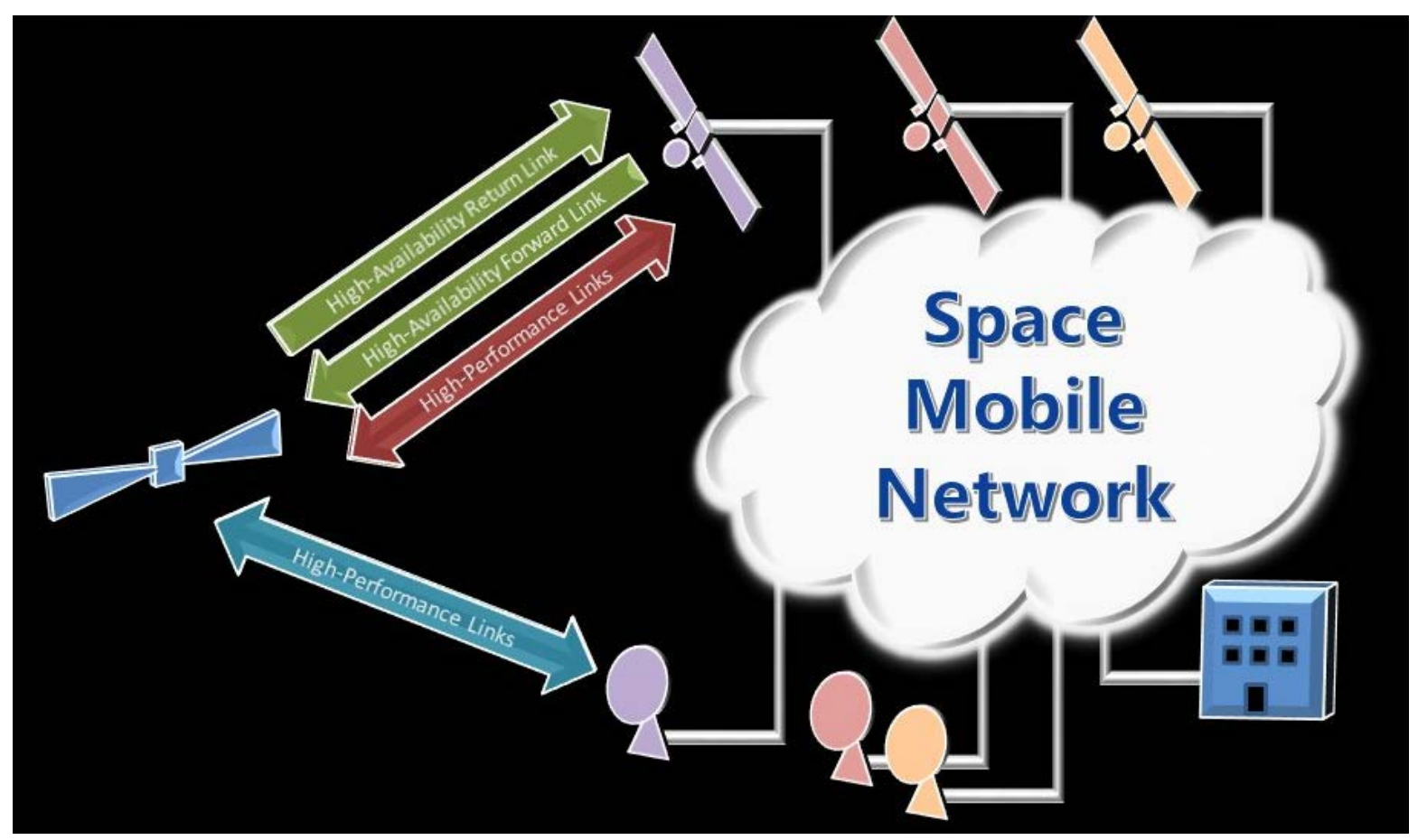

Figure 1 - Space Mobile Network Key Features

Figure 1 illustrates the key features of the Space Mobile Network. A significant difference of this view from the historical NASA space communications view is the depiction of the network as a "network cloud” with access points. This depiction has been common for terrestrial networks for some time, but the networks supporting robotic and human space exploration have continued to mainly be viewed and operated as point-to-point link providers. This view implies the ability to route or forward data between any two end points with connectivity to the network. In terrestrial networks, this functionality is provided by the ubiquitous Internet Protocol (IP) and its suite of associated protocols. Though the IP suite works in some space applications, the dependence on full end-to-end connectivity for data delivery and prevalence of "chatty" two-way support protocols prohibits its use for all space scenarios [3]. Delay/disruptiontolerant networking (DTN), specifically the Bundle Protocol (BP), has been developed to provide the benefits of networking in space (and other challenging) environments [4]. BP provides network-layer functionality using a storeand-forward approach, providing for storage at intermediate nodes when the next hop is unavailable.

As the evolution of the terrestrial internet has demonstrated, the standardization of network and link-layer protocols allows a build-up of infrastructure through the peering of provider systems. The SMN framework continues this evolution by leveraging IP and DTN for the network-layer standards and by leveraging commercial and CCSDS standards for the link- and physical-layer standards for space applications. The infrastructure to provide SMN services to future space users is expected to be comprised of global government and commercial systems. These systems, either ground stations or relay spacecraft, would provide access points to the larger network.

The terrestrial mobile network user is accustomed to having continual access to the network. Providers strive to provide coverage to all users at availability and quality-of-service levels high enough to attract and maintain customers. There are significant differences, though, between the terrestrial mobile network user and the initial SMN user. Most notable are the SMN user locations, user terminal limitations and the user's willingness to wait for full data delivery. High-availability links are a feature of the SMN, but due to the challenges involved in providing service at locations such as deep space or planetary surfaces, continuous availability is not always feasible. The SMN's highavailability links are optimized for coverage and availability, which typically limits the performance with respect to data rates and link capacity. A common approach to increasing availability is to implement multiple access systems that can provide links to multiple users. Multiple access may be provided through time, frequency or code division. A user's time slice, carrier frequency or code may be pre-provisioned such that a user can immediately receive service or a multiple-access link acquisition process may occur first. The Tracking and Data Relay Satellite System's (TDRSS) Demand Access System is an example of the former [5] and the CCSDS Proximity-1 Protocol (Prox-1) is an example 
of the latter [6]. Terrestrial mobile network systems are able to economically "over-provision" the area to allow all users to find an available link, except in extreme cases such as emergencies. These systems also maintain continuous control-channel links that can always locate users and assign parameters. This is a continuous power drain and link requirement on both the user and provider systems that is not acceptable for most spacecraft.

Space links optimized for availability will likely have traded performance in other areas. High-gain systems that will support higher data rates or reduce the user's system gain requirements are generally implemented with larger apertures with narrower beam widths and/or high-gain amplifiers. The costs measured in relay size, weight and power, or in ground station implementation and operations costs, are high, limiting deployment opportunities. Thus, these high-performance links become shared resources. Fortunately, many space missions have enough onboard storage and few stringent latency requirements that allow them to wait until the service is available. High-performance links ranging from X-band to Ka-band in radio frequency (RF) and now expanding to optical links are scheduled days to weeks in advance; this guarantees link availability in time to meet mission requirements, but leads to inefficiencies in link utilization and an inability for more rapid call-up of a high-performance link to support an unplanned science event or other occasion.

In recognition of the desire to have a more responsive method to provide high-performance links and network services, SMN introduced the concept of user-initiated services (UIS). UIS is a class of service acquisition processes in which the end user originates the service request. This differs from the current methods used for acquisition of services by allowing the service acquisition process to be carried out by standardized "machine-to-machine" communications over space links. These requests may extend beyond link access requests to requests for end-to-end data delivery [7]. UIS will enable a user platform to request services over a signaling channel embedded within any links available to the user. The high-availability links provide the most available path for UIS to request services from the network. Since most space missions and networks are unable to support an always-connected control channel due to onboard power limitations and pointing and coverage constraints, UIS solutions specific to various SMN scenarios are under development.

\section{Service Acquisition via User-Initiated Services}

Space communications operations can be described as occurring in two phases: service acquisition and service execution. Current space communications service acquisition processes are characterized by pre-planned service requests negotiated among user missions and provider network operators weeks in advance by "human-in-the-loop" systems. These requests are typically for point-to-point services and, therefore, space link resource-specific. This reduces the ability of the provider network to allocate service requests among space link resources according to optimal prevailing conditions or other criteria. In contrast, terrestrial wireless network providers implement all of the control data flows preceding service execution autonomously and hidden from end users, resulting in the delivery of user service data, such as delivering a text message to a friend or streaming internet video.

UIS automates current processes for space communications service acquisition using a request-response design pattern, with the service request generated by the user [8]. The data contents of the request message may vary based on user mission compatibility constraints, degree of platform autonomy or other considerations. However, a key distinction from current service acquisition processes is that a UIS request may be service-oriented as opposed to link resource-specific. For example, a user may specify a request to "deliver 25 gigabytes of data from the mission platform to the science operations center within two hours," or "get as much data as possible off my platform as soon as possible (to avoid overwriting the on-board data storage), and get it delivered to the science operations center within six hours." Requests specified in these terms allow the provider network flexibility to optimize allocation of the request across the set of link and network resources that satisfy the user mission service and link parameter constraints, which may be provided by government, university or commercial resources operating as a federated network.

A key architectural principle for realizing service-orientated requests in terrestrial networks involves the separation of concerns pertaining to network signaling and control data flows, which enable autonomous monitoring and control of resources, from those of the user data flows, which traverse the paths orchestrated by the signaling and control processes [9]. UIS is an emerging class of space communications service acquisition processes implemented through signaling and control protocols. In the SMN framework, signaling and control data flows will typically occur via highavailability space links. However, high-performance space links optimized for user service data flows may also carry control data. 
Under the UIS framework, the service provider pre-provisions a signaling channel to support the necessary handshaking process between the provider and any users wishing to acquire service. Once a user has secured service commitment from the provider, the actual communications of user data will take place during the service execution phase on a data channel. Service acquisition by definition always precedes service execution, and the delay between the two processes is determined by the scheduling agreement reached between the users and the provider during the service acquisition process. Users with existing service on any data channel may use it to simultaneously initiate a UIS process to acquire additional service in the future. UIS protocol facilitates the exchange of a user's request and the confirmation of provisioning of resources. Therefore, it is a service management application-layer protocol at the provider-user interface. This process must be common to all service domains in terms of procedure and messaging content in order to integrate management and operations across all elements of a federated network. However, how UIS messages are delivered across the signaling channel may vary depending on the operational environment.

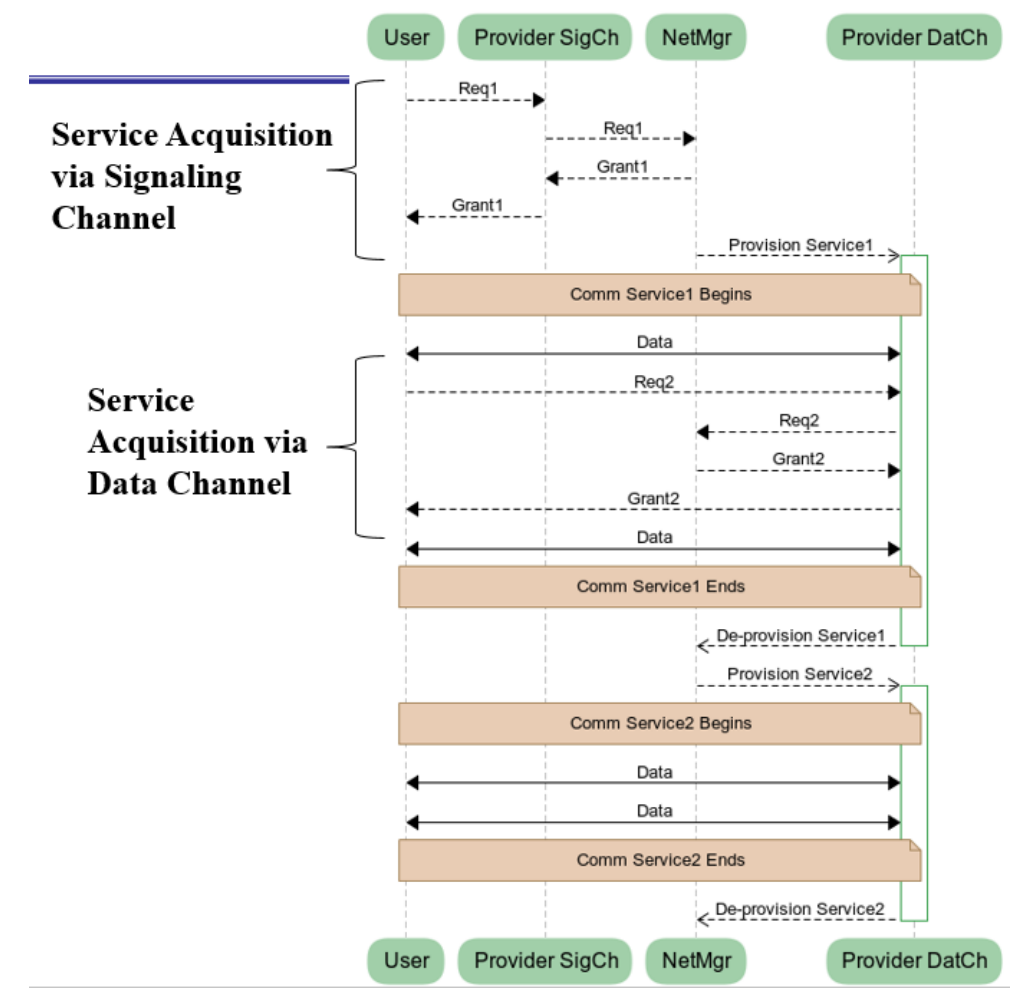

Figure 2. General UIS service acquisition process using both the signaling channel as well as the data channel for conducting the UIS handshaking process.

Under the UIS framework, a user will initiate a service request by sending a request message (REQ) to the provider over either a signaling channel or an existing data channel. A request can convey a specific desired service configuration or a range of acceptable parameters in terms of time, duration, data rate, coding, etc. In the latter case, the provider may narrow down the list of options when granting service. Upon receipt of a REQ message, the provider will determine, per network management decision, whether to grant the user's request. The request can be granted as is, granted with a more restricted parameter set, denied implicitly by lack of response and therefore result in a user time-out, or denied explicitly by issuing a negative acknowledgement (NAK). The provider's response can be issued over the signaling channel or via an existing data channel. A UIS service acquisition process is nominally a two-way handshake (positive ACK) process with user time-out. Each user must receive a confirmation from the provider within a time-out period to proceed to service execution. The provider may also have the option to explicitly cancel a prior grant to de-conflict with late-arriving, higher-priority requests. Furthermore, the provider may release/cancel a provisioned resource upon determination that service has not been utilized for a certain period of time after the beginning of the service execution phase. A one-way handshake process may be used in cases of high communications delays with a high likelihood of a granted request. In rare circumstances, a one-way handshake process might be 
considered to support off-nominal events, like a spacecraft emergency. In such a scenario, a high-detectionprobability, low-false-alarm signaling mechanism is typically used, and the user assumes the provider will correctly receive its request and grant service without further confirmation.

The specific channels available and UIS process and protocols will differ as dictated by communications constraints in different scenarios and environments. Wherever possible, UIS protocol messages and processes can be supported over different links. For example, the same UIS handshaking process and messages can be supported over a TDRSS demand-access channel or a Proximity-1 hailing channel, with the same messages carried over the different physical and link layers.

The following sections provide examples of how UIS could be implemented within different scenarios.

\section{Direct-to-Earth (DTE) Scenarios}

\section{A. Low-Earth Orbit (LEO) DTE}

Direct-to-Earth communications operations in the low-Earth orbit (LEO) regime are typically characterized by long periods with no communications access due to geometrical line-of-sight constraints between ground assets and mission platforms. Many ground link resources are concentrated near the Earth's polar regions to support sunsynchronous and other polar orbits common to weather and other Earth-observing satellites. Due to phase differences between typical LEO orbital periods and the rotational speed of the Earth, access to ground link resources for missions in lower inclination orbits is less frequent. When ground stations are in view, there are still limitations to how many missions may be supported simultaneously. However, DTE link resources, such as ground-based omni-directional or electronically steerable phased arrays, may enable more responsive DTE communications and higher availability.

In the absence of high-availability communications links, LEO missions must implement sufficient on-board autonomy to sense and respond to scientific or engineering events of interest that require responsiveness on time scales less than the greater of the expected maximum pre-scheduled inter-link access time or network service acquisition and execution time. Examples of such on-board autonomy may include increasing instrument data sampling rates in response to a transient solar flare or changing spacecraft state into a safe mode if an engineering parameter exceeds its pre-defined limit. Currently, systems and operations concepts must be designed to accommodate this increase in data volume without the ability to acquire more high-performance links in a timely fashion.

Two possible scenarios involving UIS and DTE links are described. In both scenarios, a scientific or engineering event precipitating the need for service acquisition occurs at some point between pre-planned, high-performance service events. The first scenario, depicted in Figure 3, involves ground-based, high-gain link resources only. In this scenario, a UIS request for future, high-performance link services is inserted into a pre-scheduled service channel, assuming the user needs cannot be fully satisfied during this contact. The request for future services should be dispositioned and a response provided to the user platform within the contact access window.

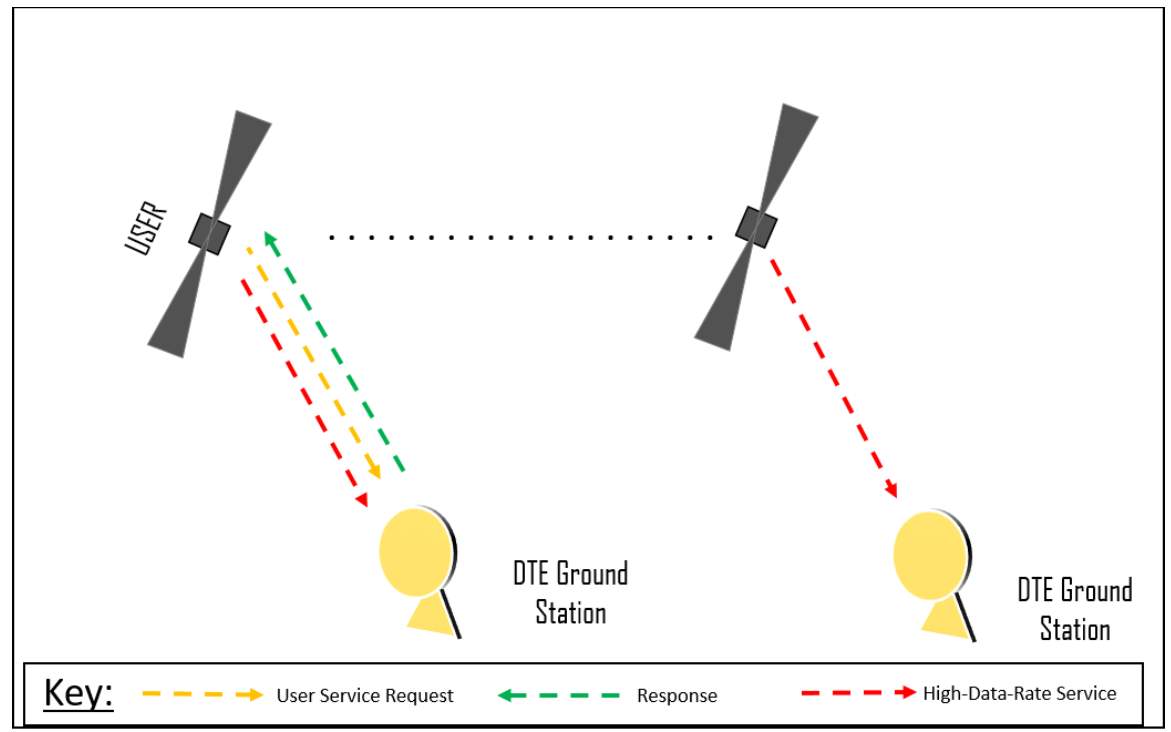

Figure 3. UIS request for Future DTE High-performance Link Access during a Pre-Scheduled DTE Contact 
The second scenario, depicted in Figure 4, involves use of higher-availability ground link resources, such as omnidirectional or electronically steered phased arrays, in order to request access to higher-performance, high-gain link resources.

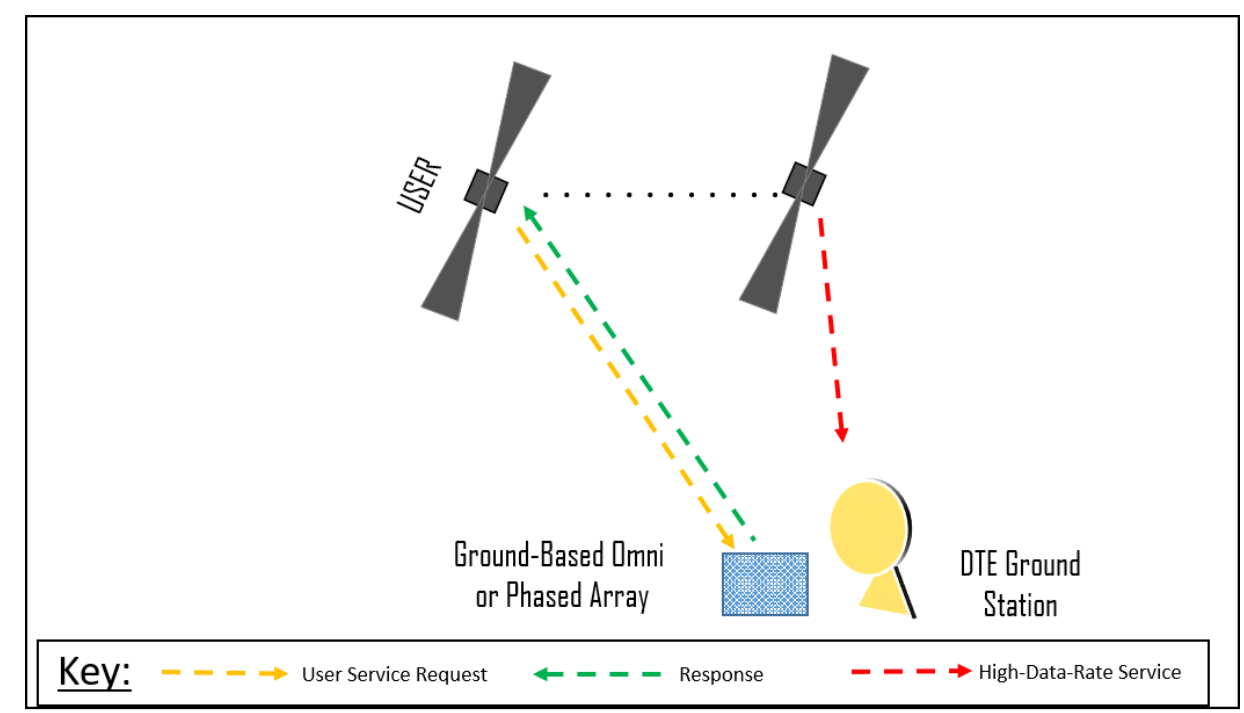

Figure 4. UIS Request for DTE High-Performance Link Access Using Higher-Availability Ground Link Resource

If possible, the service request could be granted within the same contact. The utility of this scenario is greatly enhanced by adaptive link technologies, such as variable modulation, coding and data rates, and DTN. For example, a typical LEO access window is constrained by the line of sight between the ground link resources and mission platform, and it may last ten minutes. Within that window, the range between the platform and ground link resource may vary by an order of magnitude. Adaptive link technologies would enable higher data rates as the range decreases. For a service request with a given data volume, this reduces the time required for service execution, which then forms a deadline constraint on the preceding high-availability service acquisition process. DTN protocols ensure reliable data delivery despite intermittent link availability [4]. This has three main benefits. First, the network-layer functionality allows the platform to determine where data should be sent when it gets to the ground station without any a priori knowledge at the ground station, and the store-and-forward nature of DTN provides automated rate buffering for any rate mismatches or disconnections over the end-to-end data delivery path. Second, it allows for a relaxation of constraints on link performance requirements because data delivery reliability is handled at the networking layer instead of at the link layer or application (file) layer. Third, the data from the mission platform is fractionated into bundles, which are generally smaller in data volume as compared to other common space protocol data units. These benefits combine to increase the quantity of viable access windows. From the UIS perspective, a scenario involving higher-availability ground link resources combined with adaptive link and DTN technologies may significantly improve both the responsiveness and efficiency of the space communications network.

If the service request cannot be granted during the same contact, the UIS service acquisition process could still be completed by scheduling a new or modified contact to follow. This subsequent contact could again be provided by any provider's asset if it were part of a peering or federated service infrastructure.

\section{B. Deep-Space DTE}

For deep-space communications, the UIS framework can be supported by an explicit signaling channel implemented via a beaconing system, or via an opportunistic multiple spacecraft per aperture (O-MSPA) technology [10]. The beacon approach, shown in Figure 5, is particularly suitable for missions on long cruise or during extended periods of inactivity such that it is more economical to use a low-complexity beacon signal that can be detected reliably on the ground without utilizing the 34-meter or 70-meter antennas. A preliminary beaconing concept has been tested as early as 1999 with the Deep Space-1 (DS-1) spacecraft. [11]. Due to the robustness of the beacon system, several deep-space missions (e.g. the Mars Science Laboratory and Juno) began utilizing a limited set of distinct beacon frequencies tones during critical entry, descent, and landing and orbit-insertion phases to indicate the spacecraft's 
operational states. For UIS, however, a message-oriented process is envisioned using sequences of tone "alphabet" to encode the UIS service request and confirmation messages. While all beaconing in deep space has been one-way only so far, an uplink beacon, when feasible, can be added to complete the two-way handshake. For assets deployed in the proximity of a high-coverage area such as the Mars region, where multiple spacecraft are tracked on a daily basis, OMSPA and multiple uplink per antenna (MUPA) technologies [12] can provide suitable downlink as well as uplink signaling channels for a UIS service acquisition process. The exact signaling format on the link layer and waveform could again be a simple tone-based alphabet or CCSDS-formatted telemetry; depending on the resource availability, UIS messages could be captured and processed either in the close-loop receiver with timely frame/packet content extraction and delivery to network management, or be recorded in the open-loop recorder and post-processed. Both approaches could be used depending on the desired turn-around time and ground resource availability. The common UIS service acquisition protocol is advantageous for missions operating across multiple space regimes (such as in the near-Earth regime immediately after launch followed by the lunar or deep-space regime) because the same operational procedure applies in terms of how communications services are acquired.

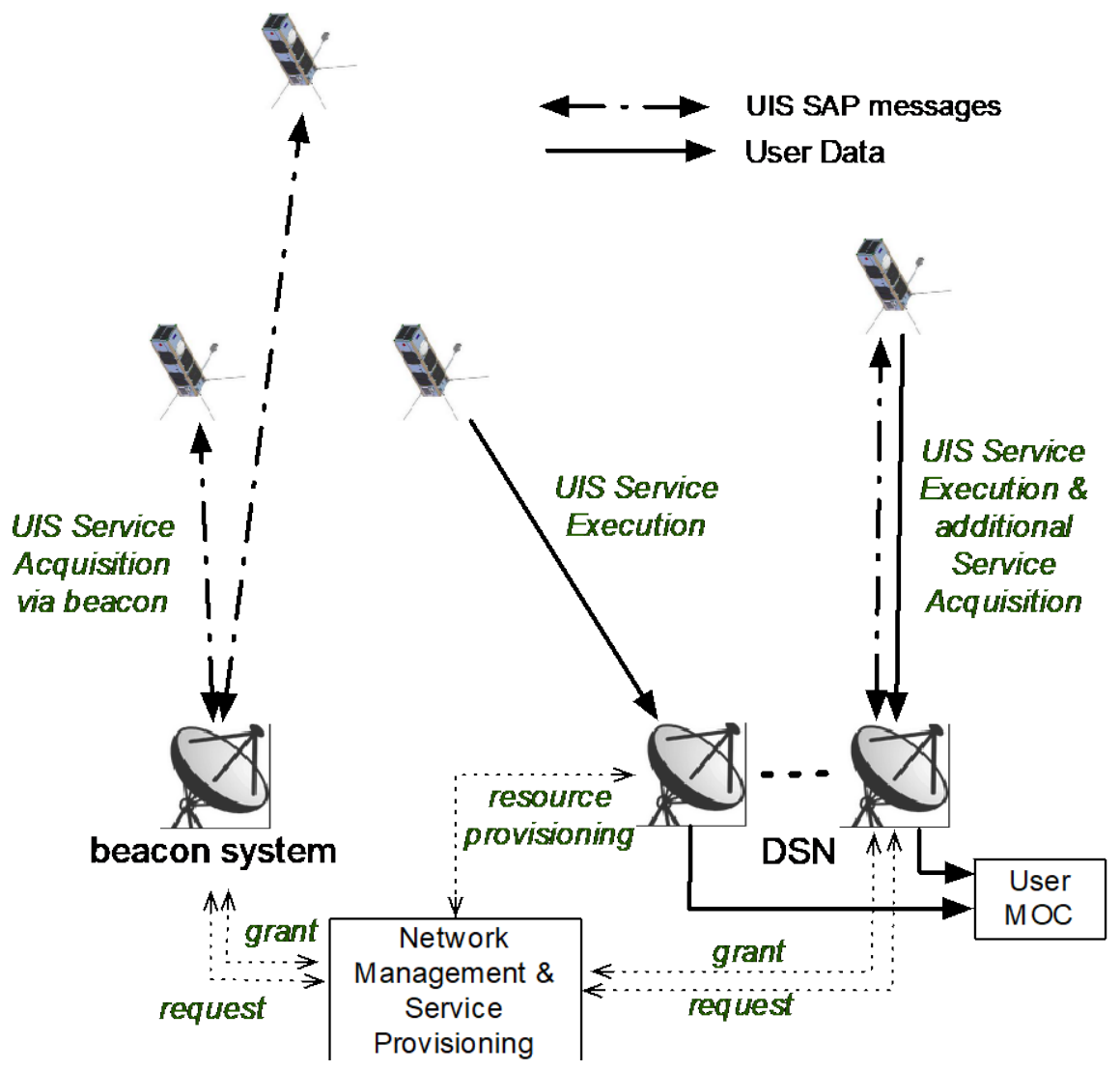

Figure 5. UIS Framework for Deep-Space Multiple Access Communications with Beacon

\section{Beyond LEO/Near Earth}

As mentioned in the previous section, with respect to supporting deep-space missions in the early-cruise phase, missions beyond LEO and out to the Moon and to the Sun-Earth L1 and L2 Lagrange points can be supported with shared apertures at sizes of 18 meters or less. The smallest antenna size that can still maintain the desired links would be preferred since that would have the widest beam width and therefore, the most coverage area for a particular distance from Earth. For example, all users on the near side of the Moon or in lunar orbit could be supported with MSPA and engage UIS to request a high-performance link at any time. The distinct advantage at these distances is that the delays of no more than a few seconds would allow two-way UIS protocols to finalize a service acquisition protocol quickly and potentially bring up a high-performance link immediately. 


\section{Relay Scenarios}

\section{A. Earth Relay}

Previous papers have extensively described the Earth relay scenario combined with the hybrid Earth relay/DTE scenario [7]. Earth relays are able to provide full orbital coverage and could feasibly provide continuously available links. There is also the greater likelihood of multiple providers among international and commercial partners that could provide the total infrastructure. One provider's system may provide the high-availability link used for the UIS service acquisition process that acquires a different provider's high-performance link. For example, a high-availability RF link may be used to schedule access on a different relay's optical link. Similar to the TDRSS Demand Access System, high-availability links could be pre-provisioned with enough simultaneous user capacity to allow each user's high-availability link service execution to begin immediately without any per-use service acquisition process.

\section{B. Mars Relay}

The current baseline of the Mars Relay Network (MRN) is based on pre-scheduled operations. However, due to the flexibility that is already designed into the primary link-layer protocol, the CCSDS Proximity-1 Space Link, channel synchronization and coding, and physical-layer protocol suite (Prox-1), the MRN can be extended easily to support UIS service acquisition. Prox-1 uses a multiple-access link acquisition process, or "hailing process," to establish a link. Figure 6 shows UIS operations utilizing the same hailing channel as its signaling channel. In this scenario, the user (the surface assets) will initiate a hailing sequence to establish a temporary connection with the provider (the relay orbiter) in order to exchange service request and handshake messages. This initial exchange on the hailing channel completes the UIS service acquisition phase. That phase is followed immediately, if available, or later by the separate Prox-1 hailing process, triggered from either the orbiter or the surface asset to kick-off the service execution phase and establish a data channel. The application-layer UIS process remains essentially the same except that the signaling mechanism is enabled by the Prox- 1 hailing process. If a user desires to acquire additional service during an existing data session, it can send the UIS message over the Prox-1 data channel, as well. This operational scenario is completely in agreement with the general UIS framework described in earlier sections.
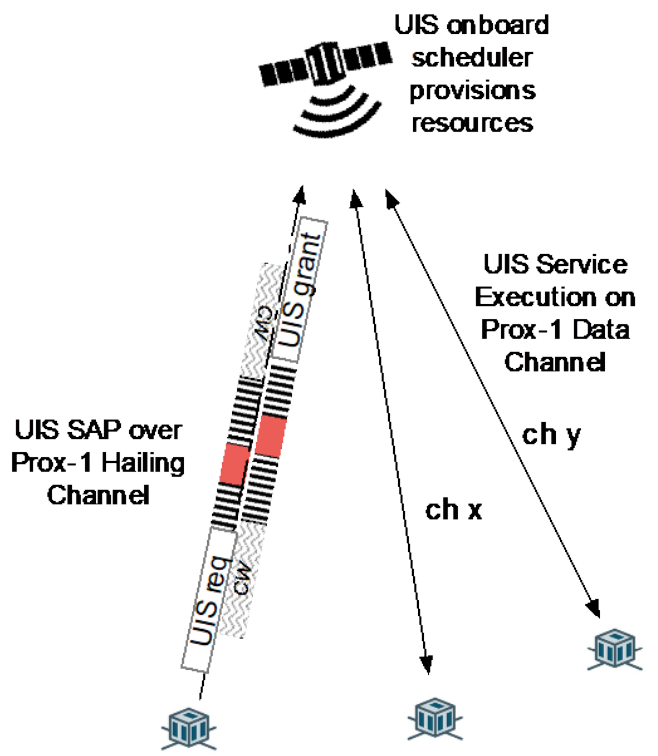

Figure 6. UIS Framework for Multiple-Access Proximity Relay Networks

\section{DTE Relay Hybrids}

\section{A. LEO}

Generally speaking, high-performance DTE links impose fewer burdens on the user platform than highperformance geosynchronous relay links. This is due to the smaller distance between the platform and link resource and the common use of un-steered, ISO-gain antennas on the platform as opposed to range distances of several tens of thousands of kilometers and gimbal-pointed antennas on the platform typically required for high-performance 
geosynchronous links. DTE communications architectures are anticipated to have lower responsiveness due to less access to ground link resources in typical LEO orbits as compared to geosynchronous Earth relay scenarios with global coverage.

However, more responsive DTE mission concepts could be enabled by high-availability links provided by space relays without the increased user burden imposed by high-performance relay links. A hybrid SMN infrastructure combining the availability of relays with the high performance of DTE links would capitalize on the best attributes of both. It is already common for missions to have low-rate relay capabilities for health and safety, so the UIS process would take place over this existing capability. UIS would allow a user to request and receive service from whichever capable assets are available, and DTN would allow data to flow to the desired destination no matter which access point was used to connect to the larger network.

In this scenario, the UIS service acquisition process occurs mainly over the high-availability, possibly preprovisioned relay links, while the high-performance links are DTE or, if capable of meeting the service request, relay links. This scenario is depicted in Figure 7 below.

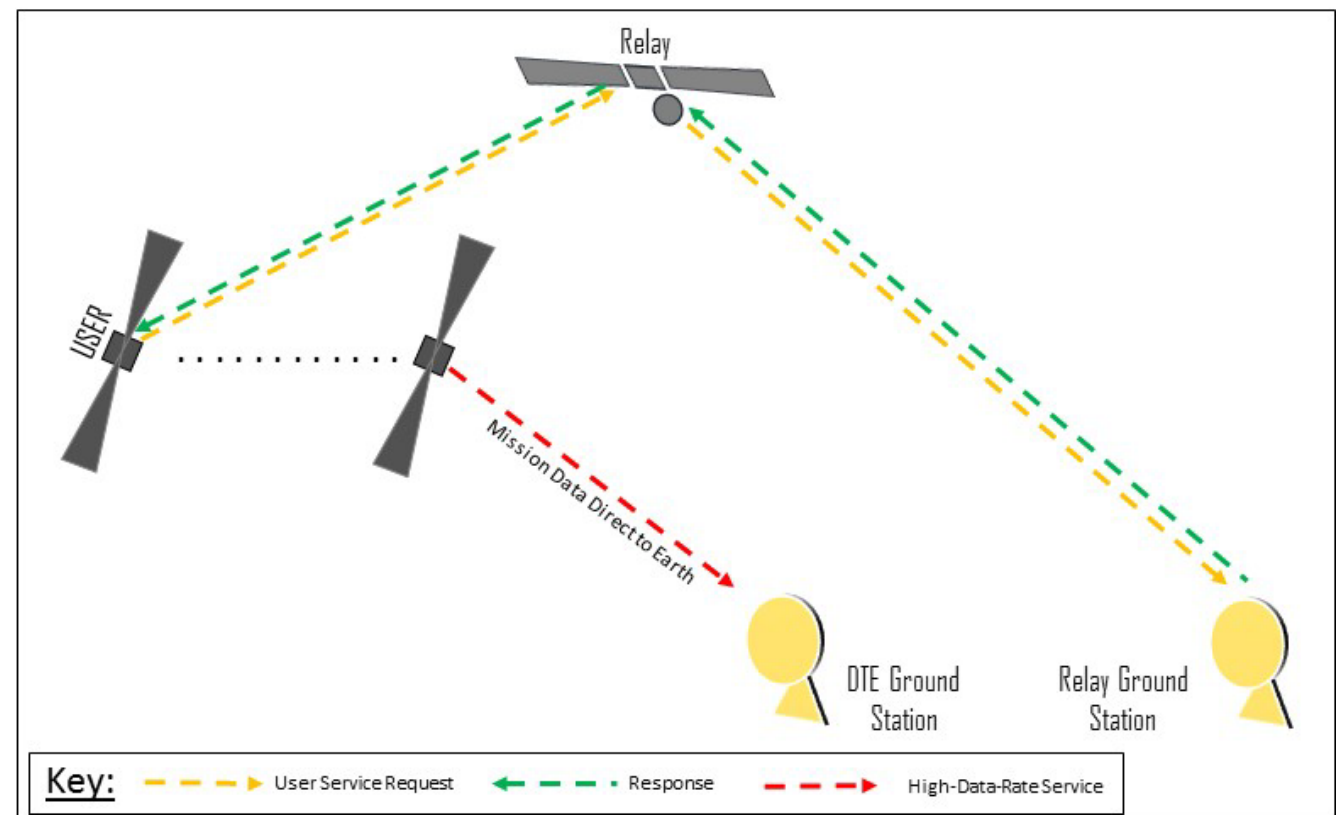

Figure 7. High-Performance DTE Service Acquired Through UIS over High-Availability Relay Service

\section{B. Distributed Space Systems}

An interesting SMN case is the Distributed Space System (DSS), in which multiple spacecraft operate within a single link coverage area. For the DSS, communications between an individual spacecraft and the provider system may function through another node within the DSS, which acts as the SMN access point. The DSS node could also expand beyond being solely a data access point to providing the link for the UIS signaling traffic. In this way, the DSS node functions as a relay in a hybrid relay/DTE system. Individual nodes may be able to schedule up DTE support or better relay support via this node of the same DSS.

For gateway nodes, those that bridge the spacecraft constellation with the ground network over DTE links, a beacon system or MSPA/MUPA can support UIS operations with the ground infrastructure. For much larger constellations with many assets spread over hundreds of thousands of kilometers or more, DTE/direct-from-Earth links will most likely be the primary communications system, and the same MSPA/MUPA approaches can apply very effectively. If the node functioning as the connection to Earth is being supported on a high-performance DTE link, more complex UIS messaging may be used than what is possible over a beacon or lower-rate multiple-access scheme. Once again, DTN provides the increased flexibility for link selection and intermediate data storage, enabling satisfied user requests with a sparser infrastructure.

\section{Lunar}

The plans for "long-term exploration and utilization" of the Moon include the build-up of multiple spacecraft landing on and orbiting the Moon [13]. This is expected to be a collaborative effort combining human and robotic 
spacecraft provided by NASA, along with commercial and international partners. The scenarios described in this paper combine for this scenario. Individual missions could be supported with DTE links from the lunar vicinity, and these missions could also become relays for other missions as part of a DSS. The inclusion of network-layer protocols for all data flows will initially facilitate the use of any link as a possible path toward data delivery. The addition of UIS concepts will provide the means for a scalable infrastructure that can be responsive to the wide variety of potential missions expected.

\section{Conclusion}

Through the recasting of the space communications architecture as a mobile network with different access points, the Space Mobile Network concept strives to enable mission operations concepts to remain consistent even as the missions move between the environments near Earth out to deep space. In general, a mission could perform low-rate data transfers and network service requests over high-availability links, higher-rate transfers over high-performance links that can be brought up more responsively to service requests, and networked communications to allow data flows between any two nodes connected to the same network. The specifics for how those links and service requests are performed will differ between environments, but that can be treated as the equivalent of "lower-layer" differences such that mission applications can be developed with defined interfaces to these underlying functions.

Key next steps are to continue to refine the concepts and terminology, define functional and performance requirements for target environments and operational concepts, and develop, model, and demonstrate protocols and implementations. Demonstrations via small satellites [14] or other test platforms, such as the SCaN Testbed [15] will also serve to identify requirements and opportunities. SMN also drives new requirements for spacecraft position, navigation and timing (PNT) systems. Other next steps include continued efforts to address meeting those new requirements and the developing new SMN-enabled PNT capabilities [16]. The components can be implemented separately and build upon each other, allowing for a phased deployment of the Space Mobile Network.

\section{Acknowledgments}

This work is in support of the NASA's Space Communications and Navigation Program Office. The work carried out at the Jet Propulsion Laboratory, California Institute of Technology, was under a contract with NASA. C. J. Roberts would like to thank professors Tom Bradley and John Borky for their contributions in shaping the UserInitiated Services concept. D. J. Israel and C. J. Roberts acknowledge the contributions of Jacob Burke, Mark Sinkiat and Jacob Barnes in defining the UIS relay and DTE scenarios. We also acknowledge Seema Vithlani for her contributions in technical writing, technical editing and graphics development.

\section{References}

[1] D. J. Israel, G. W. Heckler, R. J. Menrad. "Space mobile network: a near earth communications and navigation architecture.” Aerospace Conference, 2016 IEEE (pp. 1-7), March 2016.

[2] D. J. Israel, G. W. Heckler, R. J. Menrad, D. Boroson, B. S. Robinson, J. Hudiburg, D. M. Cornwell. “Enabling Communication and Navigation Technologies for Future Near Earth Science Missions.” 14th International Conference on Space Operations (p. 2303), May 2016.

[3] Burleigh, S., Hooke, A., Torgerson, L., Fall, K., Cerf, V., Durst, B., ... Weiss, H. (2003). "Delay-tolerant networking: An approach to interplanetary internet.” In IEEE Communications Magazine, 41(6), 128-136, June 2003.

[4] "Rationale, Scenarios, and Requirements for DTN in Space.” Report Concerning Space Data System Standards, CCSDS 734.0 G-1. Green Book. Washington, D.C.: CSDS, August 2010.

[5] T. A. Gitlin and W. Horne. “The NASA Space Network Demand Access System (DAS).” SpaceOps 2002 Conference (p. 50), Houston, Texas, 2002.

[6] "Proximity-1 Space Link Protocol—Data Link Layer.” Recommendation for Space Data System Standards, CCSDS 211.0-B 4. Blue Book. Issue 4. Washington, D.C.: CCSDS, July 2006.

[7] C. Roberts, R. Morgenstern, D.Israel, J. Borky, T. Bradley. "Preliminary results from a model-driven architecture methodology for development of an event-driven space communications service concept.” Space Terrestrial Internetworking Workshop, IEEE Wireless for Space and Extreme Environments, Montreal, Canada, October 2017. 
[8] G. Hohpe, B. Woolf, “Enterprise Integration Patterns: Designing, Building and Deploying Messaging Solutions.” Addison Wesley 2004.

[9] E. K. Pentikousis, S. Denazis, et al., "Software-Defined Networking (SDN): Layers and Architecture Terminology, Internet Research Task Force (IRTF) Request for Comments.” 7426, ISSN: 2070-1721, January 2015.

[10] D. Abraham, S Finley, D. Heckman, N. Lay, C. Lush, B. MacNeal. “Opportunistic MSPA Demonstration \#1: Final Report,” IPN Progress Report 42-200, February 15, 2015.

[11] M. Rayman, P. Varghese, D. Lehman, L. Livesay, “Results from the Deep Space 1 Technology Validation Mission.” In Acta Astronautica 47, p. 475 (2000), 50 ${ }^{\text {th }}$ International Astronautical Congress, Amsterdam, The Netherlands, 4-8 October, 1999.

[12] D. Abraham, “Progress Toward Simultaneous Communications with Multiple SmallSats via a Single Antenna.” International SmallSat Conference, Session C-1, San Jose, California, May 1-2, 2017.

[13] Hill, B. “The Next Great Steps.” Space Policy Directive 1, 45th Space Congress, 2018.

[14] H. Shaw, D. Israel, C. Roberts, J. Burke, J. Kang, J. King, "Space Mobile Network (SMN) User Demonstration Satellite (SUDS) for a practical on-orbit demonstration of User Initiated Services (UIS).” AIAA $15^{\text {th }}$ International Conference on Space Operations, Marseille, France. May-June 2018.

[15] D. Mortensen, C. Roberts, R. Reinhart, “Automated Spacecraft Communications Service Demonstration Using NASA’s SCaN Testbed.” AIAA 15 ${ }^{\text {th }}$ International Conference on Space Operations, Marseille, France, May-June 2018.

[16] J. E. Valdez, B. Ashman, C. Gramling, G. Heckler, R. Carpenter, "Navigation Architecture for a Space Mobile Network." AAS Guidance, Navigation and Control Conference, Breckenridge, Colorado, 2016. 